\title{
Pixel super-resolution in optical time-stretch microscopy using acousto-optic deflector
}

\author{
Antony C. S. Chan, Edmund Y. Lam and Kevin K. Tsia* \\ Department of Electrical and Electronic Engineering, The University of Hong Kong, Pokfulam, Hong Kong \\ *tsia@hku.hk
}

\begin{abstract}
We present experimental demonstration of pixel super-resolution time-stretch imaging by high-speed agile-beam-steering with the use of synchronized acousto-optic deflector - enabling high-resolution imaging rate of $1 \mathrm{MHz}$ whereas relaxing the stringent requirement on extreme data acquisition.
\end{abstract}

(C) 2015 Optical Society of America

OCIS codes: $100.0118,100.6640,170.3880$

Enabling ultra-high continuous frame/line-scan rate (as high as multi-MHz and even more), optical time-stretch imaging is proven to be an unique potential solution for high-throughput image-based bioassay with high-content single-cell image information (e.g. morphological information, quantitative phase and and absorption contrast [1-3]). Operating at an ultrafast imaging rate, however, entails the need for high-speed, high-bandwidth digitizer $(>40 \mathrm{GSa} / \mathrm{s}$ sampling rate, $>10 \mathrm{GHz}$ analog bandwidth) to guarantee sufficient time-stretch image pixel resolution. Such stateof-the-art specification might prohibit the wide-spread utilization of this imaging technology for practical biomedical diagnostics. To address this challenge, we have previously proposed to adopt the concept of pixel super-resolution (SR) to time-stretch imaging with the aim of relaxing the stringent requirement on high-bandwidth digitizer whereas the high image resolution can still be preserved [4]. Common in conventional photography/imaging, pixel SR imaging is employed to circumvent the pixel-size limitation (or equivalently to improve spatial-sampling bandwidth) by fusing multiple subpixel-shifted, low-sampling-rate (LR) frames with minimal reduction in the effective frame rate [6]. The key technical challenge of adopting pixel SR technique with time-stretch imaging is to implement ultrafast sub-pixel shifts in order to maintain the high frame rate on the order of $1 \mathrm{MHz}$. In this paper, we experimentally demonstrate that such ultrahigh-speed sub-pixel shift (in the multi-MHz regime) can be achieved by synchronized high-precision beam steering through the use of acousto-optic deflector (AOD) - realizing practical pixel SR time-stretch imaging system at a single-shot line-scan rate of $1 \mathrm{MHz}$.

Fig. 1 shows the schematics of an AOD-enabled pixel-SR time-stretch imaging. A broadband pulsed laser (centered at $1064 \mathrm{~nm}$ ) is time-stretched by a dispersive fiber before feeding into the microscopic setup in free space. The pulsed

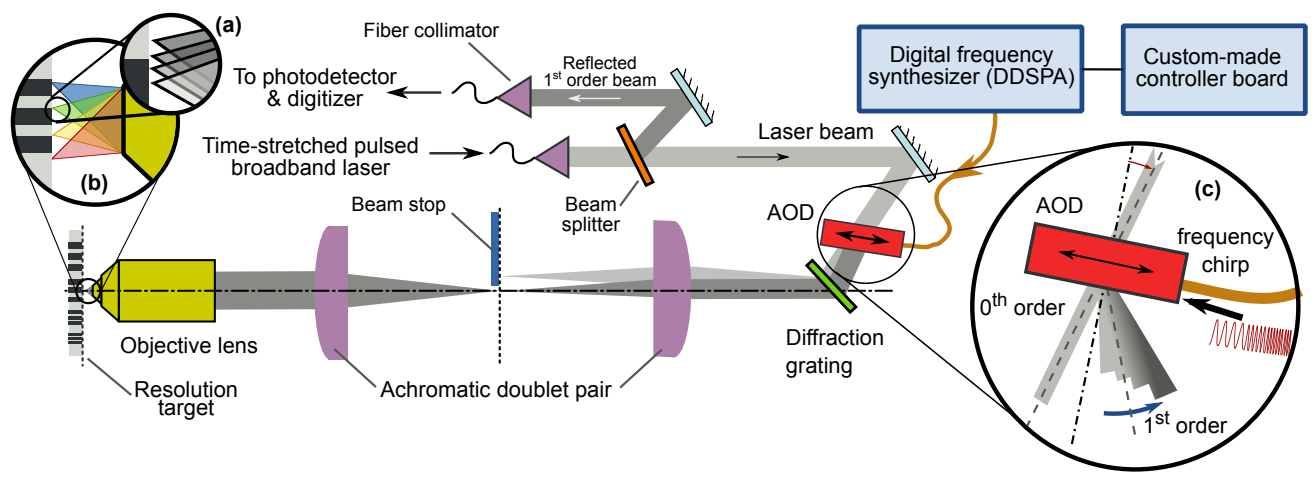

Fig. 1: Schematics of AOD-enabled pixel super-resolution (SR) time-stretch imaging. Subpixel beam steering is realized by feeding an microwave frequency chirp to the AOD. For the sake of clarity, only the laser beam at center wavelength $(1060 \mathrm{~nm})$ is shown here. (a) Subpixel-shifted laser beam of single wavelength component; (b) Zoom-in view of spectral-encoding process with multiple wavelengths; (c) exaggerated view of Bragg diffraction by the AOD. 

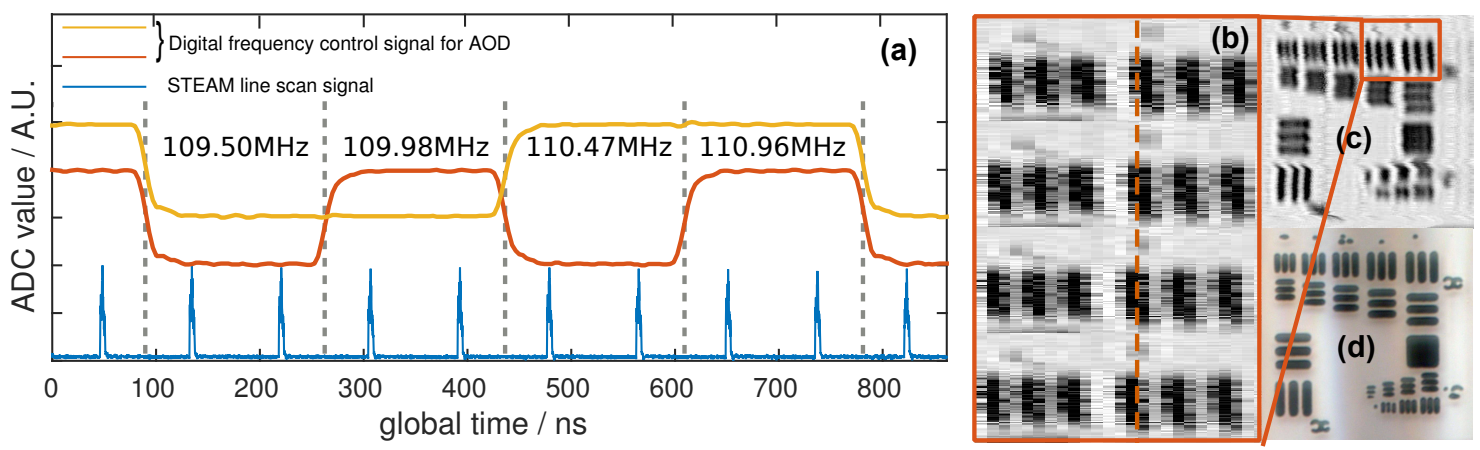

Fig. 2: Four low-sampling-rate (LR) frames are captured by precisely controlling the subpixel shift by the AOD. (a) Single-shot time-stretch image in synchronous with the beam steering signals driving the AOD (i.e. the yellow and orange traces representing the binary frequency control signals $(00,01,10,11))$. The 8 consecutive pulses are pulseaveraged to produce (b) 4 subpixel-shifted LR frames, which are then interleaved to produce (c) a pixel SR time-stretch image; (d) A bright-field image of the USAF target group 8 and 9 is captured for comparison.

beam passes through the AOD which performs agile beam-steering at the diffraction angle directly proportional to the driving acoustic frequency onto the AOD (Fig. 1a). This beam steering is solely responsible for the ultrafast sub-pixel shifts, which will be discussed shortly. The diffraction grating, positioned at the back aperture plane of the objective lens via the achromatic doublet pair, disperses different wavelengths of the laser beam to the corresponding lateral position on the object plane - a process called spectral-encoding (Fig. 1b). The reflected beam is descanned by the same diffraction grating along the same path, and is coupled into a single-mode fiber which serves as a confocal pinhole of time-stretch imaging system. Then, time-stretch-encoded signal is relayed to the photodetector and the high-speed digitizer for image acquisition. To reject the unwanted zeroth order beam from the AOD, a beam stop is placed at the image plane between the achromatic doublet pair. The field-of-view of the STEAM system is limited to $90 \mu \mathrm{m}$ govened by the total bandwidth of the laser source $(12 \mathrm{~nm})$. To match optical resolution limit at around $2.2 \mu \mathrm{m}$, a high speed digitizer with an analog bandwidth $>16 \mathrm{GHz}$, sampling rate $80 \mathrm{GSa} / \mathrm{s}$ is required. Here, we demonstrate that the same performance can be achieved from a digitizer with lower bandwidth $(8 \mathrm{GHz})$ and sampling rate $(20 \mathrm{GSa} / \mathrm{s})$ by synchronous subpixel-shift control of the AOD.

To synchronize the AOD to the home-built pulsed fiber laser, a custom-designed printed circuit board is fabricated to phase-lock the pulse output. This is to produce a set of binary frequency control signals $(00,01,10,11)$ for an digital frequency synthesizer (AA Opto-electronic DDSPA-B15b), as shown in Fig. 2a. As a result, every 2 consecutive pules encode one set of subpixel-shifted low-sampling-rate (LR) line-scan. In total, 8 consecutive pulses complete one subpixel-shift imaging cycle. To meet these requirements, the beam has to settle at a subpixel shift of $2.2 \mu \mathrm{m} / 4=$ $0.5 \mu \mathrm{m}$ within the pulse repetition interval of $89 \mathrm{~ns}$. This is feasible with an acoustic frequency chirp $\Delta f=0.49 \mathrm{MHz}$ with a beam settle time less than $40 \mathrm{~ns}$ [7], as shown in Fig. 2. This results in an effective line-scanning frame rate as high as $1 \mathrm{MHz}$. To reconstruct a pixel SR image from multiple LR frames, the 8 consecutive spectrally-encoded pulses are pulse-averaged to 4 LR frames (Fig. 2b), and then fused by interleaving the pixelated samples (Fig. 2c). The two dimensional LR image is captured by scanning the sample stage orthogonal to the beam steering axis with the use of a motorized actuator (Newport LTA-HL) We note that this orthogonal scanning can be replaced by active beam steering control by another AOD, or other high-speed motions such as ultrafast microfludic flow [3].

Fig. 3 shows the zoom-in view of the pixel SR time-stretch image, in comparison with the same region captured by the selected LR frame. It is clear that pixel SR is able to recover the feature of Elements 5 and 6 (E5 and E6), which correspond to a spatial resolution down to $2.2 \mu \mathrm{m}$. Judging from the line profile of the high-sampling-rate (HR) image, we verify that the image resolution is not limited by the sampling rate $(80 \mathrm{GSa} / \mathrm{s})$ and analog bandwidth $(16 \mathrm{GHz})$ of the digitizer. In contrast, when the digitizer is down-graded (20 GSa/s sample rate, $8 \mathrm{GHz}$ analog bandwidth), the LR frame appears to be highly pixelated and the fine image feature is lost. Such loss is caused by the unfavorable subpixel position at which the LR frame is registered, that fails to record the fine features. Pixel SR manages to restore the lost signal bandwidth by multiple subpixel-shift acquisitions, thus guarantees that at least one LR frame can partially register the fine features. Unlike the conventional imaging concept, the significance here is that the sub-pixel timestretch image shifts is performed at an ultrafast rate (i.e. in the $\mathrm{MHz}$ regime) and with the timing precision as short as sub-nanoseconds (see Fig. 2). This is made possible by the presented high-speed synchronized agile-beam steering with the use of AOD. The image contrast of Element 5 ( $\sim 28 \mathrm{ADC}$ steps) is just below the contrast of corresponding 
(a)

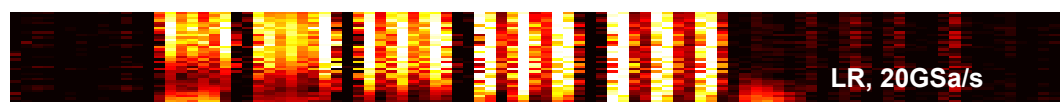

(b)

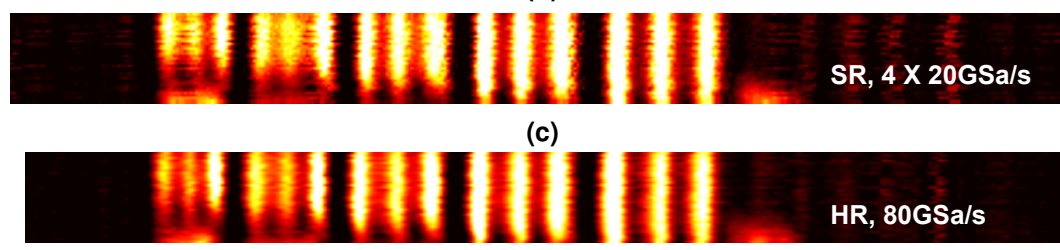

(d)

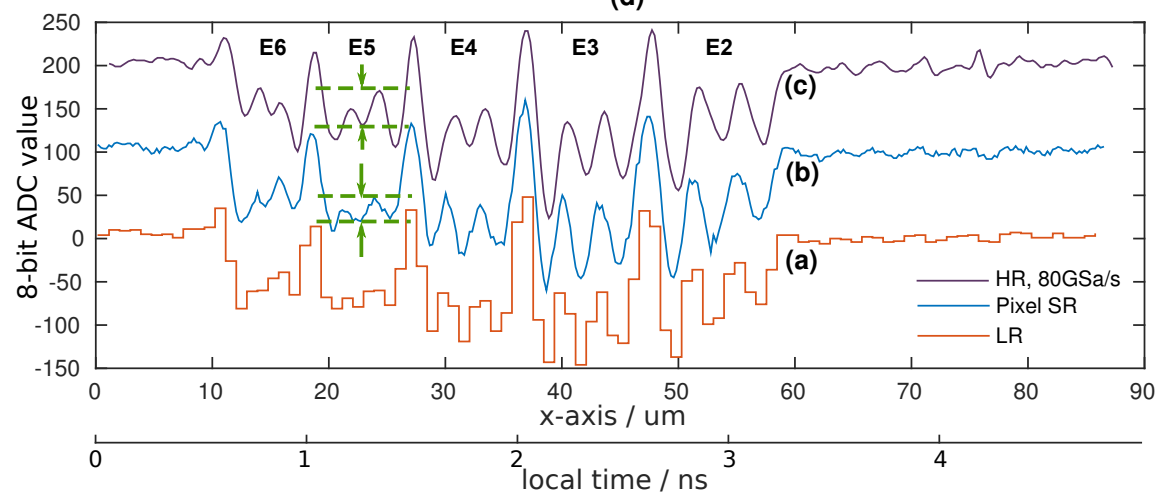

Fig. 3: Close up view of USAF target Group 8, showing an improved contrast of pixel SR time-stretch image over LR image. (a) An LR frame acquired at $20 \mathrm{GSa} / \mathrm{s}$ sampling rate, $8 \mathrm{GHz}$ analog bandwidth. (b) Pixel SR image generated from four subpixel-shifted LR frames; (c) High-sampling-rate (HR) image acquired at $80 \mathrm{GSa} / \mathrm{s}, 16 \mathrm{GHz}$ analog bandwidth; (d) line profile showing an improvement of contrast, particularly in Elements 5 and 6 (E5 and E6).

feature ( 39 ADC steps) in a high-sample-rate (HR) image (Fig. 3d) due to a lower analog bandwidth. Nevertheless, this seemingly-lost bandwidth can be recovered by image sharpening technique, present in all advanced pixel SR algorithm [6].

In summary, we have demonstrated pixel SR time-stretch imaging enabled by agile-beam-steering with the use of a synchronized AOD, at a high-speed sub-pixel shift cycle in the MHz regime and with a sub-nanosecond timing precision. This proof-of-principle demonstration (effective pixel SR line-scan rate of $1 \mathrm{MHz}$ ) shows promising application of time-stretch imaging to high-throughput and high-content image-based cellular bioassay, particularly in the format of microfluidic flow, without resorting to the state-of-the-art high-speed back-end data acquisition.

This work is partially supported by grants from the Research Grants Council of Hong Kong Special Administrative Region, China (HKU 717911E, HKU 720112E, 17207714 and 102009399), ITS/090/14 Innovation and Technology Support Programme (Tier 3) and the University Development Fund of HKU.

\section{References}

1. K. Goda, K. K. Tsia, and B. Jalali, Nature 458, 1145-9 (2009).

2. A. K. S. Lau, T. T. W. Wong, A. C. S. Chan, E. Y. Lam, K. K. Y. Wong, and K. K. Tsia, Optics in the Life Sciences 1, NW1B.4 (2013).

3. T. T. W. Wong, A. K. S. Lau, K. K. Y. Ho, M. Y. H. Tang, J. D. F. Robles, X. Wei, A. C. S. Chan, A. H. L. Tang, E. Y. Lam, K. K. Y. Wong, G. C. F. Chan, H. C. Shum, and K. K. Tsia, Scientific reports 4, 3656 (2014).

4. T. T. W. Wong, Y. Qiu, A. K. S. Lau, J. Xu, A. C. S. Chan, K. K. Y. Wong, and K. K. Tsia, 8553, 85,531P (2012).

5. T. T. W. Wong, A. C. S. Chan, K. K. Y. Wong, and K. K. Tsia, Conference on Lasers and Electro-Optics 2012 1, 1-2 (2012).

6. S. Park, M. Park, and M. Kang, Signal Processing Magazine pp. 21-36 (2003).

7. A. A. Sa, "Acousto-optics theory and application notes," Tech. rep., AA Opto-electronic (2014). 